\title{
Generation of gene disruptions by transcription activator-like effector nucleases (TALENs) in Xenopus tropicalis embryos
}

\author{
Yong Lei ${ }^{1}$, Xiaogang Guo ${ }^{2}$, Yi Deng ${ }^{3}$, Yonglong Chen ${ }^{2^{*}}$ and Hui Zhao ${ }^{1,4^{*}}$
}

\begin{abstract}
Transcription activator-like effector nucleases (TALENs) are novel engineered DNA nucleases, and have been proven to be effective for gene specific targeting in various species. Recently we reported gene disruptions in Xenopus embryos by using TALENs. Here we summarize the protocol that is used in our studies for gene disruption. This protocol covers selection of TALEN targeting sites, TALEN assembly with a modified Golden Gate method, and injection of TALEN mRNAs into Xenopus tropicalis embryos. We also provide details for detection of somatic and germ line transmitted mutations. And finally, we briefly describe establishment of knockout Xenopus lines. This protocol will facilitate broader applications of TALENs in studies of Xenopus biology.
\end{abstract}

\section{Introduction}

Xenopus tropicalis is an animal model widely used in studies for both vertebrate development and diseases because of various advantages including its vigorous fecundity, fast embryonic development, short generation period, similar morphogenetic movement as higher vertebrates and feasibility for forward genetics [1,2]. However, the barriers that impeded this model for broader use were lack of embryonic stem cell lines and the methodology of homologous recombination. Therefore it is difficult to perform specific gene targeting in Xenopus tropicalis with conventional reverse genetic methods. Such obstacles have been recently overcome by engineered endonuclease tools, the zinc finger nucleases (ZFNs) [3] and the transcription activator-like effector nucleases (TALENs) [4,5]. In particular, TALENs have been proven to be very effective for gene disruption in Xenopus as well as other animal models [5-15]. In this article, we will describe how to perform specific gene disruption with TALENs in Xenopus tropicalis embryos, and eventually establish knockout Xenopus tropicalis line.

\footnotetext{
*Correspondence: zhaohui@cuhk.edu.hk; chen_yonglong@gibh.ac.cn

${ }^{1}$ School of Biomedical Sciences, Faculty of Medicine, The Chinese University of Hong Kong, Shatin, New Territories, Hong Kong, P. R. China

${ }^{2}$ Key Laboratory of Regenerative Biology, Guangzhou Institutes of

Biomedicine and Health, Chinese Academy of Sciences, Guangzhou 510530, P. R. China

Full list of author information is available at the end of the article
}

\section{Transcription activator-like effector nucleases}

TALENs are novel engineered nucleases for gene disruption and have been successfully applied in a variety of organisms and cell types including plant [14], Drosophila [10], C. elegans [13], zebrafish [9,11], Xenopus [4,5], rat [12], livestock [6], human somatic cells [7], and human pluripotent stem cells [8]. TALENs consist of two distinct domains, an engineered DNA-binding domain derived from the transcription activator-like effector (TALE) that provides the capability and specificity for binding target DNA sequence $[16,17]$, and a non-specific restriction endonuclease Fok I domain fused to the C-terminal of TALE that confers the nuclease activity of TALENs [18] (Figure 1A).

TALEs are proteins secreted by Xanthomonas plant bacteria. These proteins bind to promoters of their target genes in host cells and activate expression of plant genes in favor bacterial infection [16,17]. A TALE protein typically consists of a translocation $\mathrm{N}$-terminal domain, a nuclear localization signal, C-terminal domain, and a central repeat domain. The central repeat domain indeed provides DNA binding specificity of a TALE protein. Each repeat unit consists of 34 amino acids, and their sequences are almost identical except for two amino acids at the 12th and 13th positions. These two amino acids which determine the binding specificity of TALE proteins to DNA bases are called repeat-

\section{Biomed Central}




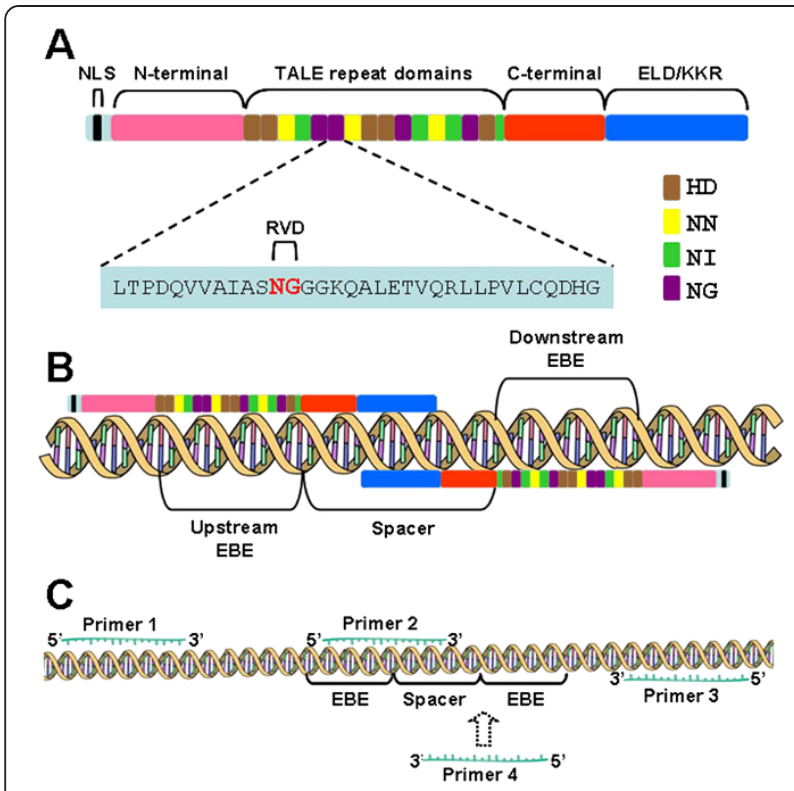

Figure 1 Schematic diagrams of TALEN structure and PCRbased assay for detection of mutagenesis. (A) Schematic drawing of TALEN structure. TALEN architecture was optimized for both zebrafish and Xenopus. Each TALEN monomer consists of a nuclear location signal (NLS), 152 amino acids deletion N-terminal, 63 amino acids from C-terminal, the TALE repeat domains, and modified Fok I nuclease domain ELD/KKR. Each TALE repeat unit consists of 34 amino acids, in which the amino acids at positions 12 and 13 are called 'repeat-variable di-residues' (RVDs). The RVD determine binding specificity to DNA bases following the code that NG, NI, HD, and NN respectively recognized thymine, adenine, cytosine and guanine. (B) Schematic diagram illustrating binding of TALENs to their targeted DNA sites. Each monomer of a TALEN pair recognizes and binds DNA via upstream or downstream EBE individually. Two Fok I nuclease domains ELD and KKR dimerize and function as endonuclease, generating DNA double strands break (DSB) at spacer between the two EBE sites. (C) PCR assay determining indel mutations induced by TALENs. The DNA fragment coving two EBE sites were amplified with Primer $1 / 3$, the amplicons were then subcloned into pMD18-T using TA cloning. Primer 1/3 were employed to check insertion of targeted sequence after TA cloning. Primer 2 covered the joint region between upstream EBE and the spacer. When indel mutations were amplified in the spacer region, no amplicons would be generated by Primer 2/3. The corresponding plasmids were then sequenced to verify TALEN-induced mutations. Primer $1 / 4$ may also be introduced to this PCR assay for detection of some mutations close to the downstream EBE site, which could not be captured by Primer 2/3. Not drawn to scale.

variable di-residues' (RVDs). NI, NG, HD and NN are known to preferentially recognize adenine (A), thymine $(\mathrm{T})$, cytosine $(\mathrm{C})$, and guanine $(\mathrm{G}) /$ adenine $(\mathrm{A})$, respectively (Figure 1A). Following this code, a given DNA sequence can theoretically be recognized by an assembled array of central repeats of TALE proteins.

Effective endonuclease activity of TALEN requires dimerization of two monomers that recognize individual DNA target sites. The Fok I domains on the two monomers designed to bind to adjacent DNA target sites will form a dimer, in turn become activated, generating DNA double-strand break (DSB) at the spacer region between two EBEs (Figure 1B). So far, no clear evidence indicates that TALEN binding to its target sequence is context dependent, but natural TALE binding site always begins with a thymine. Moreover, the off-target effects of TALENs have not been well studied yet, and should be addressed in detail by research community in the future.

\section{TALEN EBE selection and Golden Gate assembly}

As mentioned above, TALENs are effective tools for gene disruption; the key point for the application of TALENs is fast and efficient assembly. Currently, there are various methods for TALEN assembly, such as Golden Gate assembly [7], PCR-based modular assembly [19], FLASH assembly [20], as well as some commercially available approaches including GeneArt ${ }^{\circ}$ Precision TALs (Life Technologies) and TALEN $^{\mathrm{sm}}$ (Cellectis bioresearch). Among them, Golden Gate assembly has been proven to be easy, fast and effective, therefore suitable for common laboratories. We chose Golden Gate strategy for TALEN assembly in our studies. The principle of Golden Gate assembly is based on type IIS restriction enzymes that can cleave DNA out of their binding site [21]. In addition to the original report [7], we made two constructs, pCS2-TALEN-ELD/KKR, which are feasible for microinjection in both zebrafish and Xenopus embryos. We shortened both $\mathrm{N}$ - and C-terminal of TALE backbone, and replaced wild type Fok I with ELD/KKR. ELD and KKR are both derived from the wild type Fok I and can form a heterodimer to reduce potential off-target effects [22]. Our modified Golden Gate TALEN system has been proven to be suitable and effective in Xenopus embryos [5].

Previous reports indicated natural TALE binding site always begins with a thymine and its central repeat domain ends with a half repeat unit (0.5 repeat) $[16,17]$. In line with this view, the crystal structure of TALE also suggests that thymine is necessary for its recognition and binding to DNA, although few reports showed TALE could still bind to its target sequence without the thymine [23]. The TALE architecture we chose is $\Delta 152 \mathrm{~N}-\mathrm{RVDs}-+63 \mathrm{C}$. It has been successfully used for gene disruptions [24-26], and for genome editing in zebrafish embryos more recently [27]. The length of EBE and the spacer were set differently in various studies $[7,24,26,28]$. We routinely assemble TALENs harboring 15-20 repeats as such setting showed good DNA binding specificity and DNA disruption efficiency in Xenopus embryos [5]. The spacer between two EBE sites is 14-17 bps in our TALEN system.

In this TALEN mutagenesis protocol, we always select TALEN EBEs that follow a thymine. The RVDs of HD, NI, and NG preferentially bind to specific nucleotide C, $\mathrm{A}$ and $\mathrm{T}$ respectively, but $\mathrm{NN}$ is able to recognize both 
G and A [24]. Other RVD NK and NH recently were reported to have higher binding specificity to G, however, TALE reporters harboring either NK or NH showed less activity than NN $[29,30]$. Therefore currently NN is still the most commonly used RVD for recognizing G. In our studies, we tried to avoid G on the target DNA sequence in order to reduce potential off-target effects caused by the ambiguity of NN. There are also two webbased programs public available (http://taleffectors.com/ tools, and https://tale-nt.cac.cornell.edu/node/add/talen) for TALEN EBE selection.

\section{DNA Repair after double strand break induced by TALENs}

After TALE target selection, we assemble TALE repeats into pCS2-TALEN-ELD/KKR vectors. TALENs with wide type Fok I can form homodimers that may cause off-target effects. ELD/KKR are modified Fok I nuclease domains each of which carries three point mutations. TALENs harboring ELD and KKR were proven to have less offtarget effects and higher efficiency because of the obligatory heterodimer formation [22]. Upon dimerization of ELD and KKR, a pair of TALENs that binds to adjacent EBEs at a locus of interest is expected to cleave DNA double-strand at the spacer, generating DNA double-strand break. The DSB will activate DNA repair mechanism of either nonhomologous end joining (NHEJ), or homologous recombination (HR) if the proper DNA templates exist [18]. The repair of DSB by NHEJ results in small insertions or deletions (indels) at the cleaved site, leading to disruption of gene function. Repair of DSBs by HR allows integration of exogenous sequences at target site.

\section{Microinjection of TALEN mRNAs into Xenopus embryos}

The protocol for TALEN assembly will be described in the section of Experimental procedure. After we obtain TALEN constructs that target a DNA sequence of interest, these two TALEN plasmids will be linearized by NotI (NEB) and the mRNAs for microinjection will be transcribed in vitro using the linearized DNA as templates (Ambion). After purification, quality and integrity of the synthesized mRNAs will be checked by denature agarose gel, and concentrations will be determined by spectrophotometer. We normally take $500 \mathrm{pg}$ mRNA/ embryo as a trial dose for injection. The ovulation of female frogs, in vitro fertilization, embryo manipulation and microinjection were described below. Alternatively one can follow the protocol in the Harland Xenopus tropicalis website (http://tropicalis.berkeley.edu/home/).

\section{Evaluation of mutagenesis rate induced by TALENs} The somatic mutations in the injected embryos are examined at 48 hours after injection. Five injected embryos were randomly pooled and the genomic DNA was extracted for further PCR assay [5], and usually four embryo pools were used for this assay. To access the frequency of NHEJ-induced mutagenesis at the target sites, two pairs of primers were employed for this assay (Figure 1C). Primer $1 / 3$ can bridge the entire two EBE regions, and primer $2 / 3$ can link the spacer region and the downstream EBE region. PCR is performed with primers $1 / 3$ using the extracted genomic DNA as template. The amplicons will be cloned into vector pMD18$\mathrm{T}$ (Takara) or other equivalent vectors by TA cloning, colonies will be examined by colony PCR using primer pairs $1 / 3$ and $2 / 3$, respectively. In case primer $1 / 3$ generate PCR fragment, but primer $2 / 3$ fail to do so, it will suggest that the colony harbors mutation, and such colonies will be further examined by DNA sequencing. Mutagenesis rate is defined as the ratio of mutant colonies to total colonies [5].

The mutation rate $=\frac{\text { Number of mutational colonies }}{\text { Number of total positive colonies }}$

It should be pointed out that high-dose of TALEN injection may cause defects during embryonic development, revealed by curled body axis or microcephaly. To assess the possible toxicity of TALENs, we also calculate the ratio of malformation and dead embryos at $48 \mathrm{hr}$ after microinjection. Such defective embryos will normally die around metamorphosis.

\section{Evaluation of germ line transmission}

The germ line transmission is essential for establishment of a gene knockout frog line. In addition to evaluation of somatic mutations, the rest of injected embryos will be cultured to adult frog for examining germ line transmission of mutations. The G0 frogs are mosaic, carrying various mutations in their somatic cells. However, this situation normally will not affect the germ line transmission as individual sperm or oocyte only carries one mutation. To carry out germ line transmission of mutations, individual G0 frog will mate with wild type frog after HCG stimulation. The fertilized embryos (F1 generation) will be collected and cultured to stage 41 (about $48 \mathrm{hr}$ post fertilization). Genomic DNA will be extracted from individual embryos and analyzed by PCR assay described above for accessing mutagenesis rate. If a F1 embryo carries mutation, it should be heterozygous, and theoretically approximate $50 \%$ colonies will harbor mutations. The mutagenesis positive colonies will be sequenced directly to confirm sequence alternations. The ratio for germ line transmission is defined as the number of mutated F1 embryos to the total examined F1 embryos. Sibling F1 embryos will be raised to sexual maturity. The genotyping for individual F1 frogs will be 


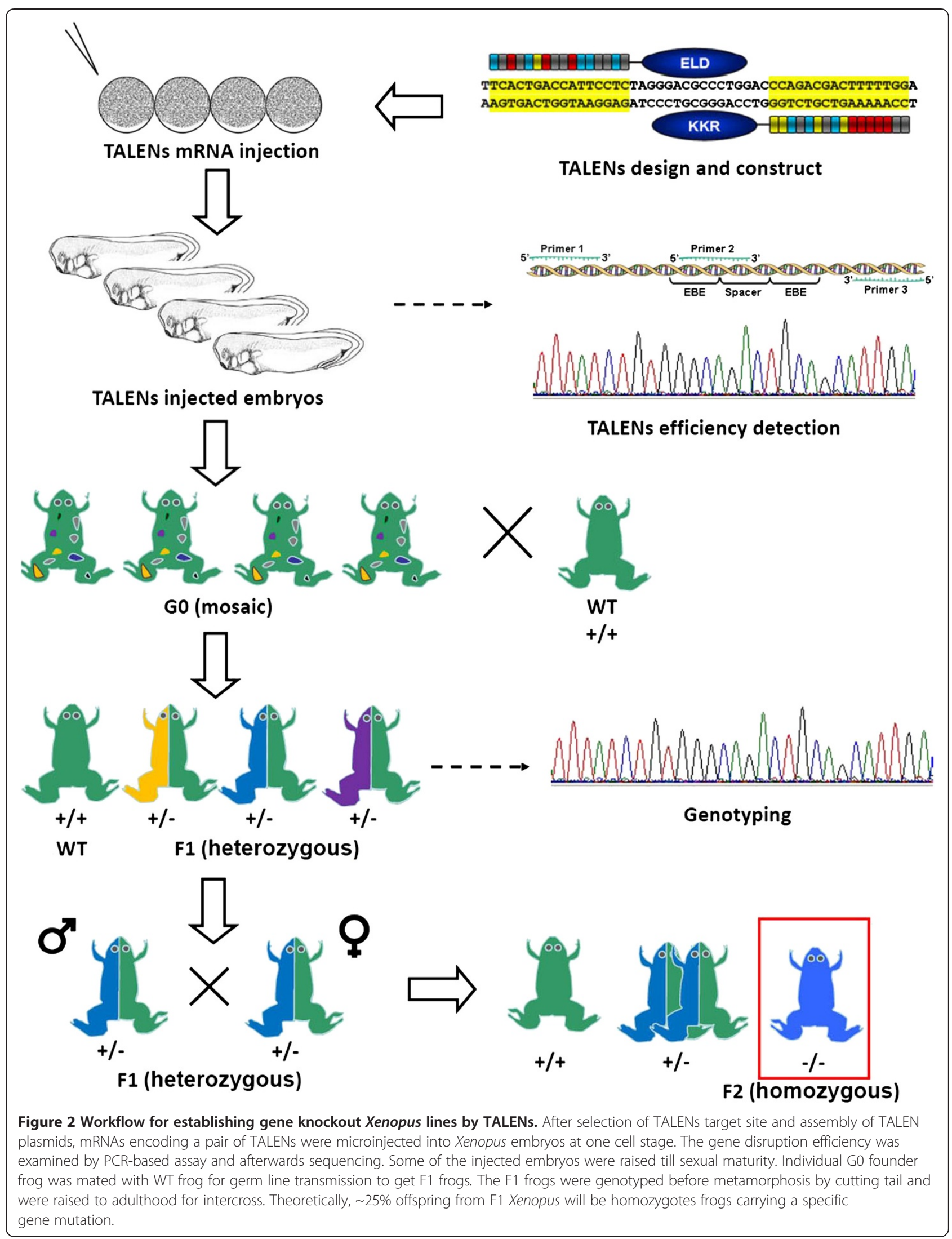


Table 1 Ligation mix in Golden Gate cloning round 1

\begin{tabular}{lll}
\hline Components & pFUS_A Mix $(\mu \mathrm{L})$ & pFUS_B Mix $(\boldsymbol{\mu L})$ \\
\hline Buffer 4 (NEB) & 1 & 1 \\
T4 Ligase (NEB) & 0.8 & 0.8 \\
Bsal (NEB) & 0.8 & 0.8 \\
RVD vectors (150 $\mathrm{ng} / \mu \mathrm{L})$ & $0.6 \times 10$ & $0.6 \times \# \mathrm{~N}-1$ \\
pFUS_A/B vectors $(150 \mathrm{ng} / \mu \mathrm{L})$ & 1 & 1 \\
ATP $(25 \mathrm{mM})$ & 0.4 & 0.4 \\
d $\mathrm{H}_{2} \mathrm{O}$ & 0 & $6-(0.6 \times \# \mathrm{~N}-1)$ \\
Total & 10 & 10 \\
\hline
\end{tabular}

performed by PCR and afterwards sequencing using genomic DNA extracted from its tail before metamorphosis (Figure 2). We often find that several F1 embryos carry the same mutation. In that case, these two frogs will be selected for mating and the fertilized embryos (F2) will be collected. In theory, $25 \%$ offspring are homozygous at the target loci. The F2 frogs will be raised and genotyped for population expansion of this knockout frog line.

\section{Experimental procedure}

Extraction and normalization of TALEN assembly vectors.

1. Recovery and extraction of TALE vectors. Obtain the Golden Gate vectors from Addgene (Cat $\# 1000000016$ or \#1000000024). Use a sterile pipette tip or inoculation loop to dip bacteria from the bacterial stab and streak bacteria onto LB Agar plates with proper antibiotics (see Reagents). On the second day, pick a single colony and culture it in LB Broth with appropriate antibiotics overnight. Extract vector DNA using a standard miniprep kit (Favorgen) ${ }^{\mathrm{a}}$, and adjust DNA concentration to 150 $\mathrm{ng} / \mu \mathrm{L}$ with TE buffer. Store plasmids DNA at $-20^{\circ} \mathrm{C}$.

2. EBE sequence selection. We routinely choose TALEN targeting sequences by the following criteria: 1 ) Thymine (T) is at position 0 and the EBE sequence follows this $\mathrm{T} ; 2$ ) the spacer sequence is around 14-17 bp; 3) minimize the number of guanine $(\mathrm{G})$ residues in EBE sequences to reduce ambiguity of RVD NN; 4) select EBEs in an exon or a junction region between intron and exon.

Table 2 Ligation program in Golden Gate cloning round 1

\begin{tabular}{lll}
\hline Cycle number & Temperature $\left({ }^{\circ} \mathbf{C}\right)$ & Time $(\mathbf{m i n})$ \\
\hline $1-15$ & 37 & 5 \\
& 16 & 10 \\
16 & 16 & 15 \\
17 & 50 & 10 \\
18 & 80 & 5 \\
\hline
\end{tabular}

Table 3 Colony PCR mix in Golden Gate cloning round 1

\begin{tabular}{ll}
\hline Components & PCR Mix $(\mu \mathrm{L})$ \\
\hline $5 \times$ GoTaq Buffer $_{2}$ & 5 \\
$25 \mathrm{mM} \mathrm{MgCl}_{2}$ & 2.5 \\
$10 \mathrm{mM}$ dNTP Mix (Fermentas) & 0.5 \\
Primer FW (10 mM) & 0.5 \\
Primer Re (10 mM) & 0.5 \\
Colony suspension & 5 \\
GoTaq DNA polymerase (Promega) & 0.5 \\
d ${ }_{2} \mathrm{O}$ & 10.5 \\
Total & 25 \\
\hline
\end{tabular}

TALENs Assembly with Golden Gate Method.

3. Assemble TALENs with Golden Gate method. The TALEN assembly in principle follows the method published elsewhere [7] except that we made two vectors pCS2-TALEN-ELD/KKR for the last step of assembly [5]. We also have optimized some reaction conditions. If the TALE length is 12-21, pick vectors for RVD 1-10 and pFUS_A, then pick RVD vector 11 up to $\mathrm{N}-1$ and pFUS_B\#N-1. If the TALE length is 22-31, pick vectors for RVD 1-10 and pFUS_A30A, RVD vector 11-20 and pFUS_A30B, then pick vectors 21 up to N-1 and pFUS_B\#N-1. The last RVD is half repeat and will be assembled in the cloning round 2.

4. Set up Golden Gate reaction mix (Cloning Round 1) listed in Table 1.

5. Perform ligation on a thermal cycler from step 4 using the following program in Table 2.

6. Treat the ligation mix with Plasmid-Safe nuclease. Add $0.5 \mu \mathrm{L}$ ATP $(25 \mathrm{mM})$ and $0.5 \mu \mathrm{L}$ Plasmid-Safe ${ }^{\mathrm{mx}}$ ATP-Dependent DNase (Epicentre) to each reaction mix. Incubate at $37^{\circ} \mathrm{C}$ for 1 hour. Plasmid-Safe nuclease can digest all unligated linear DNA including incomplete ligation products with uncompleted repeats and remaining linearized RVD vectors and pFUS vectors.

Table 4 Colony PCR program in Golden Gate cloning round 1

\begin{tabular}{lll}
\hline Cycle number & Temperature $\left({ }^{\circ} \mathbf{C}\right)$ & Time $(\mathbf{m i n})$ \\
\hline 1 & 95 & 5 \\
$2-31$ & 94 & 0.5 \\
& 52 & 0.5 \\
& 72 & 1.5 \\
32 & 72 & 10 \\
33 & 4 & $\sim$ \\
\hline
\end{tabular}




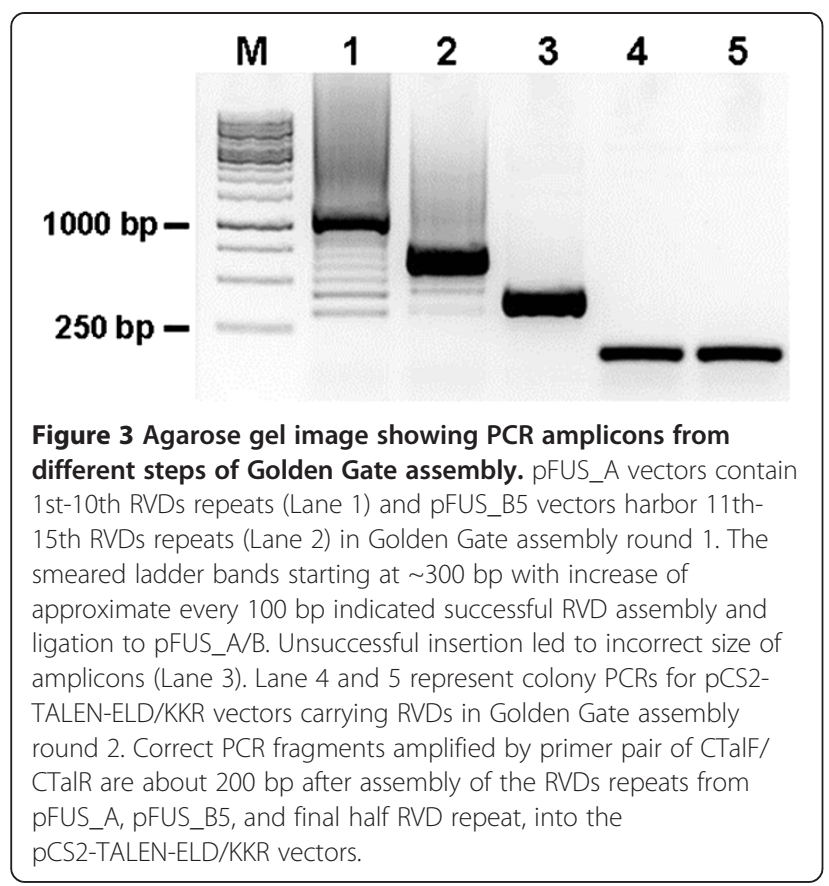

7. Transform $50 \mu \mathrm{L}$ competent cells (DH5 $\alpha$, Invitrogen) with ligation mix. Plate the transformed competent cells on Spectinomycin ${ }^{+}$LB Agar plate at $37^{\circ} \mathrm{C}$ overnight.

8. After 14-16 hr incubation, pick 7 pFUS_A colonies and 3 pFUS_B colonies. Resuspend each colony with $40 \mu \mathrm{L} \mathrm{dH} \mathrm{H}_{2} \mathrm{O}$ by vortex as PCR template. Prepare PCR mix as follows in Table 3. (The ligation efficiencies of both pFUS vectors are usually quite high. One can normally obtain positive candidate from 7 pFUS_A and 3 pFUS_B colonies).

9. Check positive clones by colony PCR with Golden Gate round 1 primers, pCR8_F \& pCR8_R (see Materials) using the following cycling program in Table 4.

10. Check the amplicons with $1 \%$ agarose gel. If the ligation is successful, colony PCR will generate smearing ladder bands and the largest band is about $1.2 \mathrm{~kb}$ for pFUS_A vectors within 10 RVDs

Table 5 Ligation mix in Golden Gate cloning round 2

\begin{tabular}{ll}
\hline Components & pCS2-TALEN Mix $(\boldsymbol{\mu L})$ \\
\hline Buffer 3 (NEB) & 1 \\
pFUS_A-RVDs vectors (150 ng/ $\mu \mathrm{LL})$ & 0.8 \\
pFUS_B-RVDs vectors $(150 \mathrm{ng} / \mu \mathrm{L})$ & 0.8 \\
Last Repeats vectors $(150 \mathrm{ng} / \mu \mathrm{L})$ & 0.6 \\
Bsmbl (NEB) & 0.8 \\
pCS2-TALEN-ELD/KKR vectors $(150 \mathrm{ng} / \mu \mathrm{L})$ & 1 \\
$\mathrm{dH}{ }_{2} \mathrm{O}$ & 5 \\
Total & 10 \\
\hline
\end{tabular}

Table 6 Ligation program in Golden Gate cloning round 2

\begin{tabular}{lll}
\hline Cycle number & Temperature $\left({ }^{\circ} \mathbf{C}\right)$ & Time $(\boldsymbol{m i n})$ \\
\hline $1-3$ & 16 & 10 \\
& 37 & 20 \\
4 & 16 & 20 \\
5 & 55 & 20 \\
6 & 80 & 5 \\
\hline
\end{tabular}

(Figure 3, Lane 1). The gel result of pFUS_B vector will also form smearing ladder bands with smaller size. The expected size was depended on the number of RVDs into pFUS_B vector (Figure 3, Lane 2).

11. Amplify the colonies harboring correct inserts in Spectinomycin ${ }^{+}$LB Broth by vigorous shaking at $37^{\circ} \mathrm{C}$ overnight.

12. Spin down the bacterial culture and perform mini prep to extract plasmid DNA (Favorgen). Adjust plasmid concentration to $150 \mathrm{ng} / \mu \mathrm{L}$.

13. Set up Golden Gate reaction mix (Cloning Round 2) listed in Table 5.

14. Incubate the reaction mix at $55^{\circ} \mathrm{C}$ for $30 \mathrm{~min}$ (BsmbI digestion). Add $0.5 \mu \mathrm{L}$ ATP $(25 \mathrm{mM})$ and $0.5 \mu \mathrm{L}$ T4 Ligase (NEB) to each reaction mix.

15. Perform ligation on a thermal cycler from step 14 using following program in Table 6.

16. Transform $50 \mu \mathrm{L}$ competent cells (DH5 $\alpha$, Invitrogen) with ligation mix. Plate the transformed E.coli on Ampicillin ${ }^{+}$LB Agar plate at $37^{\circ} \mathrm{C}$ overnight.

17. After $14-16 \mathrm{hr}$ incubation, pick 3 colonies from the selective agar plates and assess ligation (Step 13-15) by colony PCR (Step 8 ) using Golden Gate round 2 primers, CTalF \& CTalR (see Materials), with following conditions Table in 7.

18. Examine the PCR products with $1 \%$ agarose gel. The expected size of amplicons should be about 200 bp (Figure 3, Lane 4 and 5).

19. Amplify the colonies that give correct bands in Ampicillin ${ }^{+} \mathrm{LB}$ Broth at $37^{\circ} \mathrm{C}$ overnight.

20. Perform mini prep to extract DNA of pCS2-TALEN -ELD/KKR plasmids containing full RVDs

Table 7 Colony PCR program in Golden Gate cloning round 2

\begin{tabular}{lll}
\hline Cycle number & Temperature $\left({ }^{\circ} \mathbf{C}\right)$ & Time $(\mathbf{m i n})$ \\
\hline 1 & 95 & 5 \\
$2-31$ & 94 & 0.5 \\
& 52 & 0.5 \\
& 72 & 0.5 \\
32 & 72 & 10 \\
33 & 4 & $\sim$ \\
\hline
\end{tabular}


(Favorgen). Sequence the two constructs with two primers, NTalF \& CTalR (see Materials) to confirm successful assembly.

Synthesize TALEN mRNAs in vitro.

21. After sequence confirmation of assembled TALEN constructs, linearize the pCS2-TALEN-ELD/KKR constructs containing RVD with NotI (NEB). Purify the linearized DNA with Gel/PCR purification kit (Favorgen) $^{\mathrm{b}}$ according to the manufacturer's instructions.

22. Synthesize mRNAs for microinjection with mMessage mMachine SP6 kit (Ambion) using the linearized DNA (step 21) as templates. Purify the synthesized mRNA with RNeasy MiniElut Cleanup Kit (QIAgen). Check quality and integrity of the mRNA with denature agarose gel and measure RNA concentration with spectrophotometer.

Microinjection of TALEN mRNAs into Xenopus embryos and evaluation of somatic gene disruption efficiency.

23. Prior to mating, induce ovulation of female frogs with 20 IU human chorionic gonadotropin (hCG) at night before in vitro fertilizations. Inject another dose of 200 IU hCG on the next day. The female frogs lay eggs from $2 \mathrm{hr}$ to $6 \mathrm{hr}$ after hCG injection.

24. Dissect testes from a male frog, and cut testes of male frog into very small pieces and transfer them into 1xMMR (high concentration to keep sperm activity) on ice. Squeeze the ovulated female frog and collect eggs onto a petrdish. Pipet sperm mix onto eggs. Briefly mix them well and wait for 3-5 minutes. Pour 0.1xMMR into the dish to cover eggs till completion of fertilization.

25. Keep eggs at about $25^{\circ} \mathrm{C}$ throughout fertilization periods. Collect embryos and remove jelly coat from the eggs using $3 \%$ cysteine hydrochloride (in water, $\mathrm{pH}$ 7.8-8.0) after step 24.

Or follow the Harland tropicalis website to perform female ovulation, in vitro fertilization, and manipulation. (http://tropicalis.berkeley.edu/home/obtaining_embryos/ hcg/hCG.html).

26. Microinjection is performed at about $22^{\circ} \mathrm{C}$. After TALENs injection, culture the injected embryos in $0.1 \times M B S$ culture medium at $22^{\circ} \mathrm{C}$ overnight and then transfer them to $25^{\circ} \mathrm{C}$. When the injected embryos reached about stage 41 (about 48 hr post fertilization), randomly collect and pool five TALENinjected embryos, and extract genomic DNA with DNeasy blood \& tissue Kit (QIAgen) from embryo pools according to the manufacturer's instructions. Raise the rest of injected embryos to adult frogs for future germ line transmission.

27. Set up a PCR reaction to amplify the genomic region that covers two TALEN EBE sites. A pair of primers flanking the two EBE sites is employed for this PCR reaction (Figure $1 \mathrm{C}$ ). According to our experience, we recommend the length of amplicons is less than $1 \mathrm{~kb}$. Prepare PCR mix as following in Table 8.

28. After PCR reaction, check the size of PCR products, and recover the DNA with gel extraction kit (Favorgen). The purified amplicons are then cloned into pMD-18 T vector by TA cloning (Takara) using the following set-up in Table 9.

29. Incubate the ligation mix at $16^{\circ} \mathrm{C}$ for $60 \mathrm{~min}$.

30. Transform $50 \mu \mathrm{L}$ competent cells (DH5 $\alpha$, Invitrogen) with $10 \mu \mathrm{L}$ ligation mix. Plate the transformed DH5 $\alpha$ on Ampicillin ${ }^{+}$LB agar plate at $37^{\circ} \mathrm{C}$ overnight.

31. Randomly pick up at least 20 colonies for each embryo pool. Colony PCR is used to access mutagenesis rate at the targeted sites, using primer pair $1 / 3$, and primer pair $2 / 3$, respectively (Figure 4 ). If mutations are generated in the spacer region, no amplicon can be amplified using the primer pair $2 / 3$. Verify mutation by direct sequencing.

32. Raise the sibling TALENs injected Xenopus embryos till sexual maturity according to (http://tropicalis. berkeley.edu/home/husbandry/raisetads.html) or protocol published elsewhere [31]. It takes about 5 months for the injected embryos to reach sexual maturity, and longer period is also expected depending on the feeding conditions. Pigment patch appearing in frog's claws suggest maturity of male froglets.

33. When TALENs targeted frogs are ready for germ line transmission, mate a male or female G0 frog with a wild type frog. The details are described as

Table 8 PCR mix for amplification of target genomic DNA

\begin{tabular}{ll}
\hline Components & PCR Mix $(\boldsymbol{\mu L})$ \\
\hline $5 \times$ GoTaq Buffer $_{2}$ & 5 \\
$25 \mathrm{mM} \mathrm{MgCl}_{2}$ & 2.5 \\
$10 \mathrm{mM}$ dNTP Mix (Fermentas) & 0.5 \\
Primer 1/2 (10 mM) & 0.5 \\
Primer 3 (10 mM) & 0.5 \\
Genomic DNA & 0.5 \\
GoTaq DNA polymerase (Promega) & 0.5 \\
dH ${ }_{2} \mathrm{O}$ & 15 \\
Total & 25 \\
\hline
\end{tabular}


Table 9 TA cloning mix

\begin{tabular}{ll}
\hline Components & Ligation Mix $(\boldsymbol{\mu L})$ \\
\hline pMD18-T Vector (Takara) & 1 \\
Insert DNA ( 0.3 pmol) & 4 \\
Solution I & 5 \\
Total & 10 \\
\hline
\end{tabular}

follows: (http://tropicalis.berkeley.edu/home/ obtaining_embryos/natural_mating.html). Briefly prior to mating, inject both male and female frogs with 20 IU hCG at the night before natural mating and keep separately to avoid amplexus and possible fertilizations. Inject another dose of 200 IU hCG to boost reaction of both male and female frogs and place them together without disturbances on the next day.

34. Usually female frogs lay eggs from $2 \mathrm{hr}$ to $6 \mathrm{hr}$ after the second hCG injection. Keep frogs at about $25^{\circ} \mathrm{C}$ throughout the priming and natural mating periods [31].

35. Collect embryos and remove jelly coat from the eggs using 3\% cysteine hydrochloride (in water, $\mathrm{pH} 7.8-8.0)$. Culture the embryos in $0.1 \times \mathrm{MBS}$ at $25^{\circ}$ $\mathrm{C}$ until at stage 41 . Randomly collect 20 embryos, extract genomic DNA from single embryo, and leave the sibling embryos to develop to adult frogs.

36. Use the method described in step 27-31 to determine whether the F1 embryos carry mutations, and calculate the rate for germ line transmission. (number of embryos harboring mutations/total number of examined embryos). After the sibling embryos developed till stage 45, cut tail for DNA extraction to determine the genotype at the targeted loci. Select the larvae carrying open reading frame (ORF)-shift mutations and raise them to sexual maturity for the intercross ${ }^{\mathrm{c}}$.

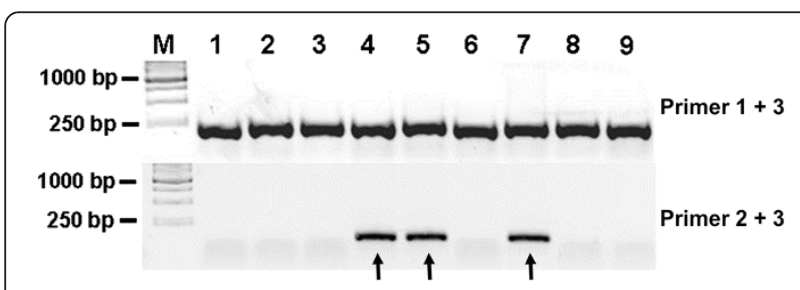

Figure 4 Colony PCR results for evaluation of gene disruption. Agarose gel showing an example of colony PCR to detect mutagenesis in ets1-TALEN injected Xenopus tropicalis embryos. The bands amplified by Primer 1/3 in upper panel ( 200 bp) indicate the colonies harbor the TALEN targeted sites. PCR assay was also carried out with Primer $2 / 3$ in lower panel. The colonies that can give upper bands but fail to give lower bands were the positive colonies carrying potential mutants. The colonies giving both upper and lower bands did not carry mutants (arrows indicated). Mutations were further confirmed by sequencing.
Table 10 Primers for Golden Gate assembly and sequencing

\begin{tabular}{ll}
\hline Primer name & Sequence \\
\hline pCR8_F & TTGATGCCTGGCAGTTCCCT \\
pCR8_R & CGAACCGAACAGGCTTATGT \\
CTalF & CGTTGACCAACGACCACCTC \\
CTalR & CTAGTTGGGATCCGGCAAC \\
NTalF & GATGACAAGGGTACCGTG \\
\hline
\end{tabular}

37. After population expansion of selected F1 adult frogs, mate the F1 frogs. About 25\% offspring from intercross of F1 adult frogs are homozygous at the targeted loci according to Mendel's law. Expand the frog population with this genome type, and maintain this Xenopus mutant line.

\section{Conclusion remarks}

In our previous studies, TALENs exhibited remarkable efficiency of gene disruption as well as broad targeting spectrum of DNA sequence in genome [5]. Those advantages make TALENs a promising gene disruption approach for specific gene targeting in various animal models including Xenopus. In this article, we described the TALENs protocol currently used in our laboratory for generating mutations in Xenopus tropicalis embryos. We listed the criteria for EBE selection and the protocol for TALENs assembling. The methods of embryo manipulation and microinjection are basically the same as those used in other laboratories. We also described a PCR-based method for detecting the targeted gene disruption efficiency and procedures for establishing a gene-specific knockout Xenopus line from TALENs injected embryos. The PCR-based evaluation method is simple and reliable. It may miss some mutations that close to the downstream EBE site. We suggest to introduce a forth primer (Figure 1C) in order to capture all the possible mutations. TALENs display high efficiency for generating somatic mutations in G0 embryos. However it is worth noting that whether TALEN can be used the

\section{Table 11 Vectors in Golden Gate Kit (This kit can be purchased from Addgene)}

\begin{tabular}{llllllllll}
\hline \multicolumn{2}{l}{ Tetracycline } & & & & & & & & \\
pNI1 & pNI2 & pNI3 & pNI4 & pNI5 & pNI6 & pNI7 & pNI8 & pNI9 & pN10 \\
pHD1 & pHD2 & pHD3 & pHD4 & pHD5 & pHD6 & pHD7 & pHD8 & pHD9 & pHD10 \\
pNN1 & pNN2 & pNN3 & pNN4 & pNN5 & pNN6 & pNN7 & pNN8 & pNN9 & pNN10 \\
pNG1 & pNG2 & pNG3 & pNG4 & pNG5 & pNG6 & pNG7 & pNG8 & pNG9 & pNG10 \\
Spectinomycin & & & & & & & & \\
pLR-NI & pLR-HD & pLR-NN & pLR-NG & & \\
pFUS_A & pFUS_A30A & pFUS_A30B & & & & \\
pFUS_B1 & pFUS_B2 & pFUS_B3 & pFUS_B4 & pFUS_B5 \\
pFUS_B6 & pFUS_B7 & pFUS_B8 & pFUS_B9 & pFUS_B10 \\
\hline
\end{tabular}


same as antisense morpholino oligonucleotides cannot be concluded before conducting comparable studies systematically. $p t f 1 a / p 48$ TALEN can induce complete loss of $x p d i p$ expression as did $p t f 1 a / p 48$ morpholino [5], but it seems that the efficiency is not as profound as that with $p t f 1 a / p 48$ morpholino knockdown in Xenopus laevis published previously [32]. We have observed similar effects with other genes. One explanation could be that the TALEN-injected embryos are mosaic and the gene disruption efficiencies vary in different embryos. And even in one embryo harboring mutations, the somatic mutations are different $[5,33]$. Moreover, TALEN cannot block functions from the maternal mRNAs. Such complexity may make it difficult to interpret phenotype induced by TALENs in G0 embryos. Therefore, we suggest that morpholinos cannot be replaced by TALENs currently. Although our protocol is for gene disruption in Xenopus embryos, it is also feasible for zebrafish with minor modifications of the setting for microinjection. With rapid growth of research on TALENs, accumulating data indicate that TALEN is an easy but robust tool for reverse genetics study. Engineered TALENs will facilitate and expand applications in various organisms for future research.

\section{Materials}

Primer List (Table 10).

Golden Gate vectors (Addgene Cat \#1000000016 or \#1000000024) (Table 11).

TALENs backbone vectors (Ampicillin)

pCS2-TALEN-ELD, pCS2-TALEN-KKR.

\section{Reagents}

T4 DNA ligase (NEB, Cat. no. M0202M)

NotI (NEB, Cat. no. R0189L)

BsaI (NEB, Cat. no. R0535L)

BsmbI (NEB, Cat. no. R0580L)

Plasmid-Safe $^{\mathrm{rm}}$ ATP-Dependent DNase (Epicentre, Cat. no. E3110K)

GoTaq DNA polymerase (Promega, Cat. no. M8295)

pMD18-T vector (Takara, Cat. no. D101A)

FavorPrep Plasmid DNA Extraction mini Kit (Favorgen, Cat. no. FAPDE001-1)

DNeasy blood\&tissue Kit (QIAgen, Cat. no. 69504)

GEL/PCR Purification Kit (Favorgen, Cat. no.

FAGCK001-1)

mMessage mMachine SP6 Kit (Ambion, Cat. no.

AM1340)

RNeasy MiniElute Cleanup Kit (QIAgen, Cat. no. 74204)

LB Broth medium (Usb, Cat. no. 75852)

LB Agar medium (Invitrogen, Cat. no. 22700-025)

Amplicillin (Sigma, Cat. no. A1593)

Spectinomycin (Sigma, Cat. no. S4014)

Tetracycline (Sigma, Cat. no. 87128)
GeneRuler 1 kb DNA Ladder (Fermentas, Cat. no. SM0311/2/3)

DH5 $\alpha$ (Invitrogen, Cat. no. 18263-012)

\section{Endnotes}

a Miniprep can be performed by equivalent commercial kit.

${ }^{\mathrm{b}}$ Gel extraction or PCR purification can be performed by equivalent commercial kit.

c It is critical to perform genome typing at the TALENs targeting sites for all adult F1 frogs prior to mating. If we find that a few male and female F1 frogs carry identical mutation, we will mate them directly. If only one frog has a particular genome type, we cross this frog with wild type frog again to expand the population.

\section{Ethical approval}

All animal experiments were performed following approval from the University Animal Experimentation Ethics Committee (AEEC) of The Chinese University of Hong Kong.

\section{Competing interests}

The authors declare that they have no competing interests.

\section{Authors' contributions}

$Y L, H Z, Y L C, X G G$, and $Y D$ wrote this methodology paper and revised it. All authors read and approved the final manuscript.

\section{Acknowledgments}

This work is supported by grants from the Research Grants Council of Hong Kong CUHK480709 and N_CUHK413/12 to H. Z., supported in part by funds from the National Basic Research Program of China (2009CB941202) and fund from the Key Project of Knowledge Innovation Program of the Chinese Academy of Sciences (KSCX2-YW-R-083) to Y. L. C, the National Natural Science Foundation of China (81200651/H0726) to Y. D. Y.L. is supported by the Graduate Studentships from The Chinese University of Hong Kong. We thank colleagues in our laboratories for helpful discussion on this project.

\section{Author details}

${ }^{1}$ School of Biomedical Sciences, Faculty of Medicine, The Chinese University of Hong Kong, Shatin, New Territories, Hong Kong, P. R. China. ${ }^{2}$ Key Laboratory of Regenerative Biology, Guangzhou Institutes of Biomedicine and Health, Chinese Academy of Sciences, Guangzhou 510530, P. R. China. ${ }^{3}$ Department of Biology, South University of Science and Technology of China, Shenzhen 518055, P. R. China. ${ }^{4}$ Shenzhen Research Institute, The Chinese University of Hong Kong, Shenzhen 518057, P. R. China.

Received: 23 January 2013 Accepted: 28 March 2013

Published: 10 May 2013

\section{References}

1. Hellsten U, Harland RM, Gilchrist MJ, Hendrix D, Jurka J, Kapitonov V, Ovcharenko I, Putnam NH, Shu S, Taher L, et al: The genome of the Western clawed frog Xenopus tropicalis. Science 2010, 328(5978):633-636.

2. Kashiwagi K, Kashiwagi A, Kurabayashi A, Hanada H, Nakajima K, Okada M, Takase M, Yaoita Y: Xenopus tropicalis: an ideal experimental animal in amphibia. Exp Anim 2010, 59(4):395-405.

3. Young JJ, Cherone JM, Doyon Y, Ankoudinova I, Faraji FM, Lee AH, Ngo C, Guschin DY, Paschon DE, Miller JC, et al: Efficient targeted gene disruption in the soma and germ line of the frog Xenopus tropicalis using engineered zinc-finger nucleases. Proc Natl Acad Sci U S A 2011, 108(17):7052-7057.

4. Ishibashi S, Cliffe R, Amaya E: Highly efficient bi-allelic mutation rates using TALENs in Xenopus tropicalis. Biol Open 2012, 1(12):1273-1276. 
5. Lei $Y$, Guo X, Liu Y, Cao Y, Deng Y, Chen X, Cheng CH, Dawid IB, Chen Y, Zhao H: Efficient targeted gene disruption in Xenopus embryos using engineered transcription activator-like effector nucleases (TALENs). Proc Natl Acad Sci U S A 2012, 109(43):17484-17489.

6. Carlson DF, Tan W, Lillico SG, Stverakova D, Proudfoot C, Christian M, Voytas DF, Long CR, Whitelaw CB, Fahrenkrug SC: Efficient TALEN-mediated gene knockout in livestock. Proc Natl Acad Sci U S A 2012, 109(43):17382-17387.

7. Cermak T, Doyle EL, Christian M, Wang L, Zhang Y, Schmidt C, Baller JA, Somia NV, Bogdanove AJ, Voytas DF: Efficient design and assembly of custom TALEN and other TAL effector-based constructs for DNA targeting. Nucleic Acids Res 2011, 39(12):e82.

8. Hockemeyer D, Wang H, Kiani S, Lai CS, Gao Q, Cassady JP, Cost GJ, Zhang L, Santiago Y, Miller JC, et al: Genetic engineering of human pluripotent cells using TALE nucleases. Nat Biotechnol 2011, 29(8):731-734.

9. Huang P, Xiao A, Zhou M, Zhu Z, Lin S, Zhang B: Heritable gene targeting in zebrafish using customized TALENs. Nat Biotechnol 2011, 29(8):699-700.

10. Liu J, Li C, Yu Z, Huang P, Wu H, Wei C, Zhu N, Shen Y, Chen Y, Zhang B, et al: Efficient and specific modifications of the Drosophila genome by means of an easy TALEN strategy. J Genet Genomics 2012, 39(5):209-215.

11. Sander JD, Cade L, Khayter C, Reyon D, Peterson RT, Joung JK, Yeh JR: Targeted gene disruption in somatic zebrafish cells using engineered TALENs. Nat Biotechnol 2011, 29(8):697-698.

12. Tesson L, Usal C, Menoret S, Leung E, Niles BJ, Remy S, Santiago Y, Vincent $A l$, Meng $X$, Zhang $L$, et al: Knockout rats generated by embryo microinjection of TALENs. Nat Biotechnol 2011, 29(8):695-696.

13. Wood AJ, Lo TW, Zeitler B, Pickle CS, Ralston EJ, Lee AH, Amora R, Miller JC, Leung $E$, Meng $X$, et al: Targeted genome editing across species using ZFNs and TALENs. Science 2011, 333(6040):307.

14. Zhang Y, Zhang F, Li X, Baller JA, Qi Y, Starker CG, Bogdanove AJ, Voytas DF: TALENs enable efficient plant genome engineering. Plant Physiol 2012, 101(1):20-27.

15. Sakuma T, Hosoi S, Woltjen K, Suzuki Kl, Kashiwagi K, Wada H, Ochiai H, Miyamoto T, Kawai N, Sasakura Y, et al: Efficient TALEN construction and evaluation methods for human cell and animal applications. Genes Cells 2013, 18(4):315-326.

16. Boch J, Scholze H, Schornack S, Landgraf A, Hahn S, Kay S, Lahaye T, Nickstadt A, Bonas U: Breaking the code of DNA binding specificity of TAL-type III effectors. Science 2009, 326(5959):1509-1512.

17. Moscou MJ, Bogdanove AJ: A simple cipher governs DNA recognition by TAL effectors. Science 2009, 326(5959):1501.

18. Urnov FD, Rebar EJ, Holmes MC, Zhang HS, Gregory PD: Genome editing with engineered zinc finger nucleases. Nat Rev Genet 2010, 11(9):636-646.

19. Zhang F, Cong L, Lodato S, Kosuri S, Church GM, Arlotta P: Efficient construction of sequence-specific TAL effectors for modulating mammalian transcription. Nat Biotechnol 2011, 29(2):149-153.

20. Reyon D, Tsai SQ, Khayter C, Foden JA, Sander JD, Joung JK: FLASH assembly of TALENs for high-throughput genome editing. Nat Biotechnol 2012, 30(5):460-465.

21. Engler C, Kandzia R, Marillonnet S: A one pot, one step, precision cloning method with high throughput capability. PLoS One 2008, 3(11):e3647.

22. Doyon Y, Vo TD, Mendel MC, Greenberg SG, Wang J, Xia DF, Miller JC, Urnov FD, Gregory PD, Holmes MC: Enhancing zinc-finger-nuclease activity with improved obligate heterodimeric architectures. Nat Methods 2011, 8(1):74-79

23. Deng D, Yan C, Pan X, Mahfouz M, Wang J, Zhu JK, Shi Y, Yan N: Structural basis for sequence-specific recognition of DNA by TAL effectors. Science 2012, 335(6069):720-723.

24. Christian ML, Demorest ZL, Starker CG, Osborn MJ, Nyquist MD, Zhang Y, Carlson DF, Bradley P, Bogdanove AJ, Voytas DF: Targeting G with TAL effectors: a comparison of activities of TALENs constructed with NN and NK repeat variable di-residues. PLoS One 2012, 7(9):e45383.

25. Dahlem TJ, Hoshijima K, Jurynec MJ, Gunther D, Starker CG, Locke AS, Weis AM, Voytas DF, Grunwald DJ: Simple methods for generating and detecting locus-specific mutations induced with TALENs in the zebrafish genome. PLoS Genet 2012, 8(8):e1002861.

26. Miller JC, Tan S, Qiao G, Barlow KA, Wang J, Xia DF, Meng X, Paschon DE, Leung $E$, Hinkley SJ, et al: A TALE nuclease architecture for efficient genome editing. Nat Biotechnol 2011, 29(2):143-148.

27. Bedell VM, Wang Y, Campbell JM, Poshusta TL, Starker CG, Krug RG 2nd, Tan W, Penheiter SG, Ma AC, Leung AY, et al: In vivo genome editing using a high-efficiency TALEN system. Nature 2012, 491(7422):114-118.
28. Mussolino C, Morbitzer R, Lutge F, Dannemann N, Lahaye T, Cathomen T: A novel TALE nuclease scaffold enables high genome editing activity in combination with low toxicity. Nucleic Acids Res 2011, 39(21):9283-9293.

29. Cong L, Zhou R, Kuo YC, Cunniff M, Zhang F: Comprehensive interrogation of natural TALE DNA-binding modules and transcriptional repressor domains. Nat Commun 2012, 3:968.

30. Streubel J, Blucher C, Landgraf A, Boch J: TAL effector RVD specificities and efficiencies. Nat Biotechnol 2012, 30(7):593-595.

31. Zhao H, Han D, Dawid IB, Pieler T, Chen Y: Homeoprotein hhex-induced conversion of intestinal to ventral pancreatic precursors results in the formation of giant pancreata in Xenopus embryos. Proc Natl Acad Sci U S A 2012, 109(22):8594-8599.

32. Afelik $\mathrm{S}$, Chen $\mathrm{Y}$, Pieler $\mathrm{T}$ : Combined ectopic expression of Pdx1 and $\mathrm{Ptf1a/p48} \mathrm{results} \mathrm{in} \mathrm{the} \mathrm{stable} \mathrm{conversion} \mathrm{of} \mathrm{posterior} \mathrm{endoderm} \mathrm{into}$ endocrine and exocrine pancreatic tissue. Genes Dev 2006 20(11):1441-1446.

33. Cade L, Reyon D, Hwang WY, Tsai SQ, Patel S, Khayter C, Joung JK, Sander $J D$, Peterson RT, Yeh JR: Highly efficient generation of heritable zebrafish gene mutations using homo- and heterodimeric TALENs. Nucleic Acids Res 2012, 40(16):8001-8010

doi:10.1186/2045-3701-3-21

Cite this article as: Lei et al:: Generation of gene disruptions by transcription activator-like effector nucleases (TALENs) in Xenopus tropicalis embryos. Cell \& Bioscience 2013 3:21.

\section{Submit your next manuscript to BioMed Central and take full advantage of:}

- Convenient online submission

- Thorough peer review

- No space constraints or color figure charges

- Immediate publication on acceptance

- Inclusion in PubMed, CAS, Scopus and Google Scholar

- Research which is freely available for redistribution

Submit your manuscript at www.biomedcentral.com/submit
C Biomed Central 